\title{
IMPLEMENTAÇÃO DE NÓS DE NÉVOA ADAPTATIVOS SOBRE O AMBIENTE DE EMULAÇÃO MININET
}

\author{
Higor Sant'Anna ${ }^{1}$; Antonio Coutinho²; \\ 1. Bolsista PROBIC, Graduando em Engenharia de Computação, Universidade Estadual de Feira de Santana, e-mail: \\ higorsantanna@ecomp.uefs.br \\ 2. Orientador, Departamento de Tecnologia, Universidade Estadual de Feira de Santana, e-mail: acoutinho@uefs.br
}

PALAVRAS-CHAVE: Névoa; Health-Care; SOFT-IoT.

\section{INTRODUÇÃO}

A Internet das Coisas (IoT) está recebendo uma grande atenção no cenário tecnológico devido a seu grande potencial e impacto em diferentes segmentos da sociedade. Atualmente, várias iniciativas IoT tem desenvolvido simultaneamente novas arquiteturas, plataformas e aplicações que resultaram na criação de sistemas IoT paralelos como pode ser visto em Bonomi (2014), Yi (2015), Dastjerdi (2016) e Openfog (2017). Isso tem tornado difícil o acesso aos dispositivos IoT e sua possível integração com aplicações.

Visando o estudo de soluções integradas e de fácil configuração nessa área, o objetivo deste trabalho foi o de validar a plataforma SOFT-IoT (Andrade, 2018) como uma maneira real de implementar nós de névoa adaptativos e reconfiguráveis, que possam manter e gerenciar diferentes serviços IoT. Assim, pesquisadores, estudantes e desenvolvedores nesta área poderiam utilizar estes componentes na construção de aplicações sofisticadas.

\section{MATERIAL E MÉTODOS OU METODOLOGIA}

No intuito de oferecer um nó de névoa adaptativo, e que disponibiliza diferentes tipos de serviços, foi empregada a plataforma SOFT-IoT (Self-Organizing Fog of Things). O SOFT-IoT é uma plataforma IoT que implementa o conceito de névoa das coisas (Fog of Things - FoT) com o intuito de disponibilizar aplicações IoT dentro do contexto da Computação em Névoa. A Figura apresenta a arquitetura SOFT-IoT proposta pelo paradigma FoT, sendo subdivido em cinco camadas:

- A Camada de Percepção também é denominada camada de dispositivo. Essa camada é composta por objetos físicos e dispositivos como os sensores, atuadores e etiquetas, que trata basicamente da identificação e coleta de informações através de sensores.

- A Camada de Rede transfere com segurança as informações dos sensores para o sistema que processará as informações.

- Na Camada de Middleware os dispositivos IoT implementam diferentes tipos de serviços. Cada dispositivo se conecta e se comunica apenas com outros dispositivos que possuam o mesmo tipo de serviço. A principal responsabilidade dessa camada é o gerenciamento de serviços e o oferecimento de conexão com alguma base de dados. 
- A Camada de Aplicação fornece serviços solicitados pelos usuários. Por exemplo, a Camada de Aplicação pode fornecer medições de temperatura e umidade do ar ao usuário que solicitar essas informações. A importância dessa camada para a IoT é que ela possui a capacidade de fornecer serviços inteligentes, com qualidade, visando atender as necessidades dos usuários.

- A Camada de Negócio gerencia as atividades e serviços gerais de um sistema IoT. As responsabilidades dessa camada são construir um modelo de negócios, gráficos, fluxogramas, etc., com base nos dados recebidos da Camada de Aplicação.

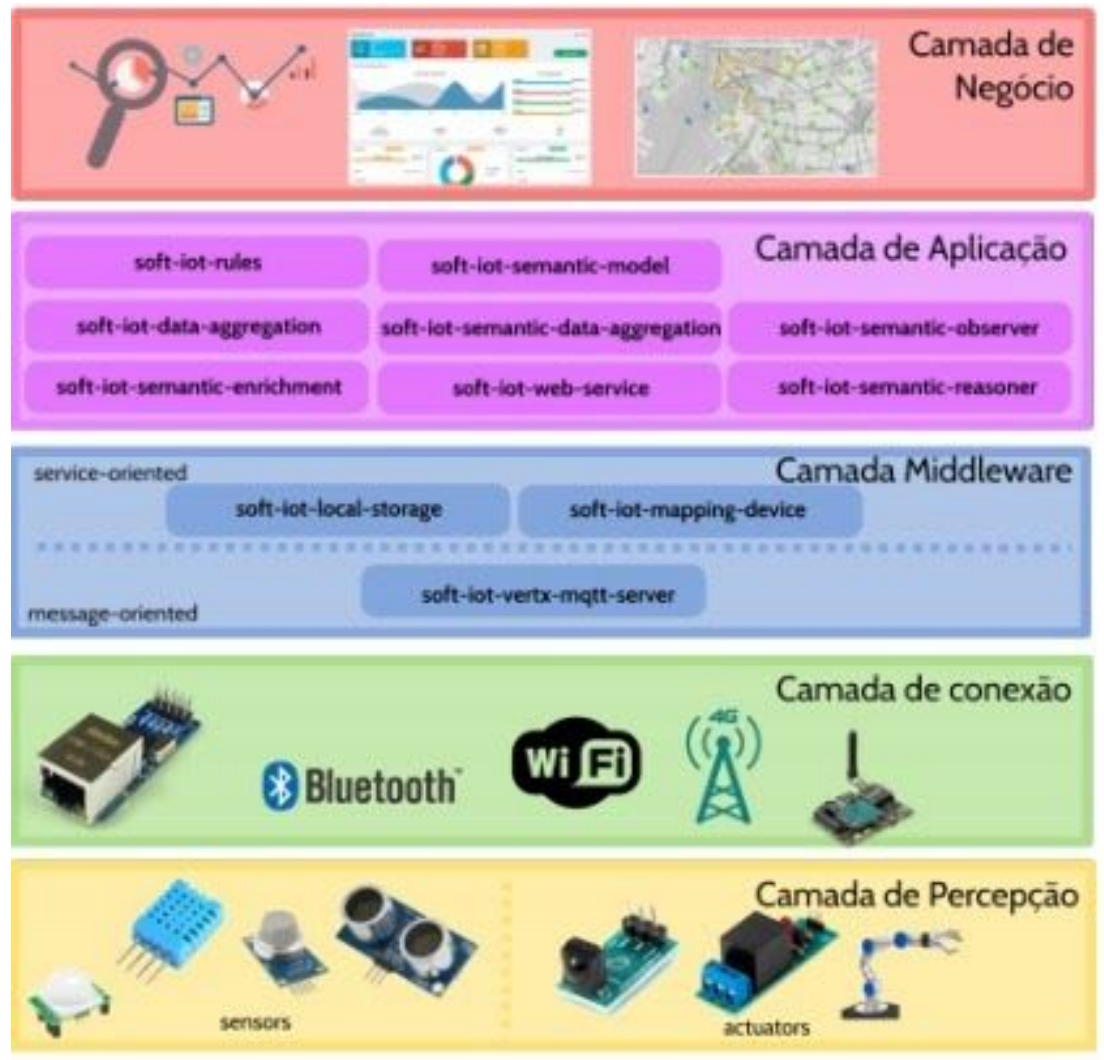

Figura 1. Arquitetura do SOFT-IoT (Andrade, 2018).

\section{RESULTADOS E/OU DISCUSSÃO}

Para validar a plataforma foi criado um estudo de caso na área de heath-care, onde uma médico quer prever em tempo real quais $k$ usuários são mais prováveis de sofrer problemas de saúde como ataque cardíaco, pressão alta e outras doenças previsíveis. O objetivo é monitorar a saúde de usuários críticos e prover assistência o mais rápido possível.

A arquitetura do experimento foi: a camada de percepção foi simulada através de quatro máquinas, cada um representando um dispositivo com vários sensores, cujo propósito é mandar informações sobre o paciente para a camada de redes, sensores esses que foram simulados através de uma aplicação; também foi utilizado quatro máquinas para a simulação das borda de rede, cada nó da borda encapsula uma instância do SOFT-IoT, e através do serviço MQTT Broker, o módulo de comunicação do SOFT-IoT, recebe dados de um (ou mais) dispositivos e calcula um top- $k$, para esse cálculo, foi criado um novo serviço dentro do SOFT-IoT, que foi chamado de Bottom 
Broker; os nós da borda passam a informação para os nós de névoa, simulados por duas máquinas,que realizam um novo cálculo de top- $k$, agora com o resultado dos top- $k \mathrm{~s}$ feitos pelo Bottom Broker, e mandam o resultado para nuvem; na nuvem o cálculo é feito mais uma vez, e o resultado final é passado para o cliente. Na Figura 2, pode ser vista a arquitetura do experimento:

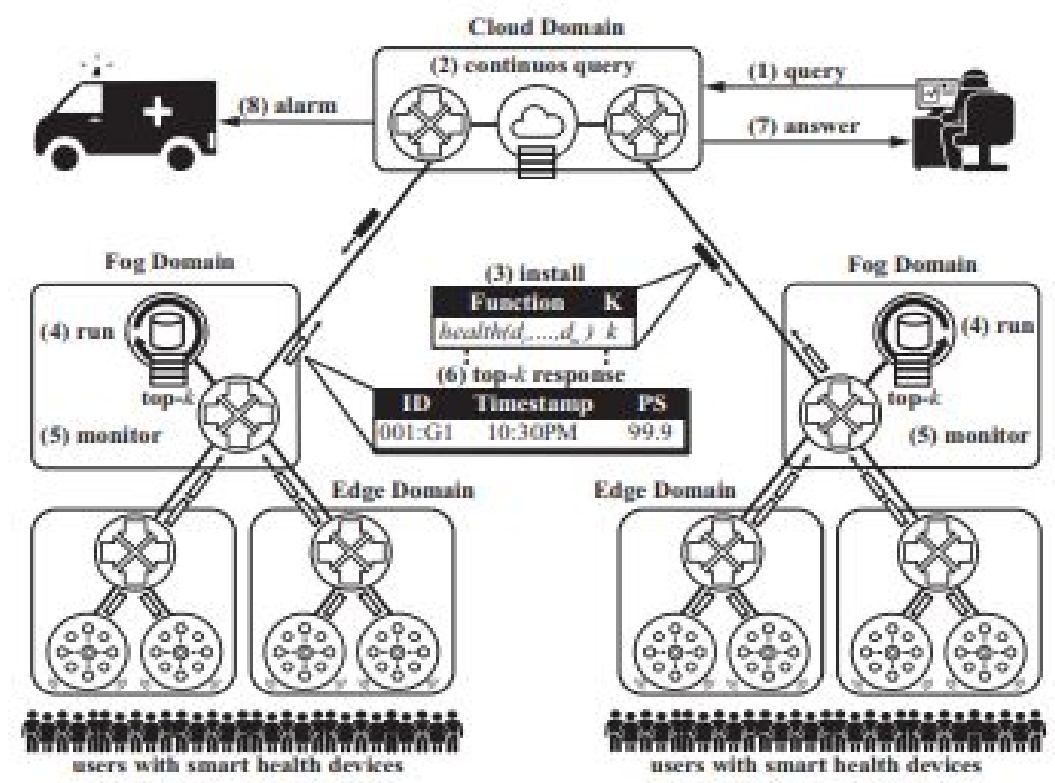

Figura 2. Arquitetura do Sistema proposta para os testes experimentais.

Foram realizados testes para avaliar principalmente: $(i)$ o tempo de resposta; e (ii) a carga exigida na execução de serviços no SOFT-IoT. Foram feitos testes com o SOFT-IoT (Método A) e comparados com a execução dos mesmos serviços, emulados da mesma forma, mas sem a utilização do SOFT-IoT (Método B). Os gráficos podem ser vistos abaixo:

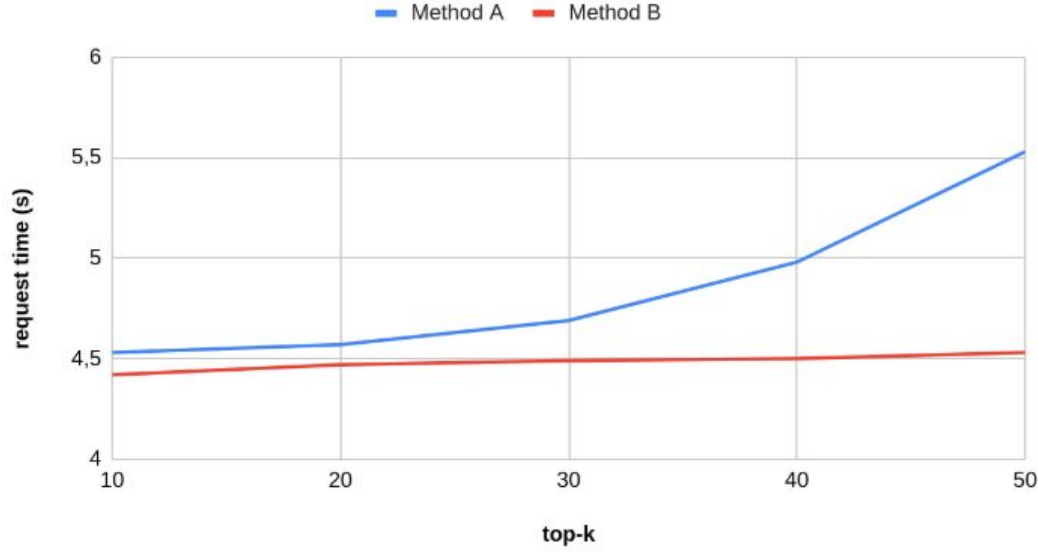

Figura 3. Comparação do tempo de resposta de cada método. 


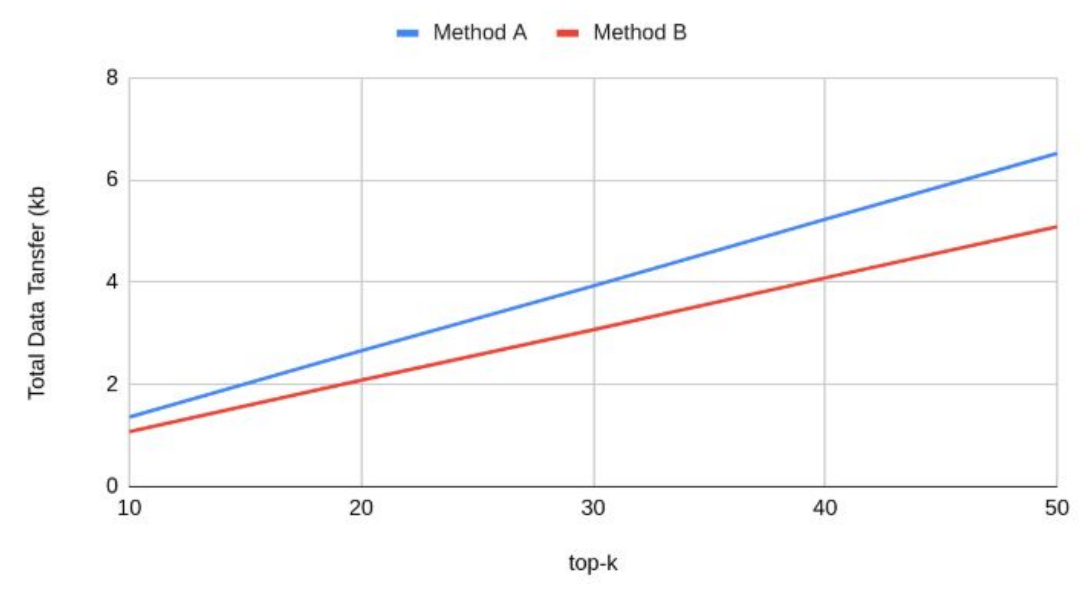

Figura 4. Comparação do total de dados transferidos de cada método.

\section{CONSIDERAÇÕES FINAIS}

Neste trabalho, foi alcançado o objetivo de criar um sistema de utilizando nós de névoa adaptativos e visualizar seus benefícios em aplicações reais. Pretende-se dar seguimento a pesquisa de duas formas: (i) uma melhora do algoritmo de top- $k$ implementado, para a melhora de performance; (ii) pela incorporação de tecnologias e arquiteturas descentralizadas que permitam melhorar o desempenho e a escalabilidade do ambiente de emulação.

\section{REFERENCIAS}

F. BONOMI, R. MILITO, P. NATARAJAN, AND J. ZHU, "Fog computing: A platform for internet of things and analytics," in Big Data and Internet of Things: A Roadmap for Smart Environments. Springer, 2014, pp. 169-186.

S. YI, Z. HAO, Z. QIN, AND Q. LI, "Fog computing: Platform and applications," in Hot Topics in Web Systems and Technologies (HotWeb), 2015 Third IEEE Workshop on. IEEE, 2015, pp. 73-78.

A. V. DASTJERDI, H. GUPTA, R. N. CALHEIROS, S. K. GHOSH, AND R. BUYYA, "Fog computing: Principles, architectures, and applications," arXiv preprint arXiv:1601.02752, 2016.

OPENFOG CONSORTIUM GROUP AND OTHERS. (2017, Feb.) OpenFog Consortium home page. [Online]. Available: http://www.openfogconsortium.org/

ANDRADE, L., LIRA, C., MELlO, B., ANDRADE, A., COUTINHO, A., GREVE, F. E PRAZERES, C.,"Do device á cloud com a Plataforma SOFT-IoT: sua infraestrutura IoT em poucas horas", em Minicursos Webmedia 2018. SBC, 2018, p. 193-240.

SERVICEMIX, APACHE. (2017, Feb.) "Apache servicemix 3. x users' guide." Apache ServiceMix community. [Online].

Disponível

em: http://incubator.apache.org/servicemix/users-guide.html. 